\title{
ORIGINAL ARTICLE Transcriptome sequencing of the choroid plexus in schizophrenia
}

\author{
S Kim ${ }^{1}$, Y Hwang ${ }^{2}$, D Lee ${ }^{2}$ and MJ Webster ${ }^{1}$
}

The choroid plexus (CP) has a key role in maintaining brain homeostasis by producing cerebrospinal fluid (CSF), by mediating transport of nutrients and removing metabolic products from the central nervous system and by responding to peripheral inflammatory signals. Although abnormal markers of immune response and inflammation are apparent in individuals with schizophrenia, the CP of these individuals has not been characterized. We therefore sequenced mRNA from the CP from two independent collections of individuals with schizophrenia and unaffected controls. Genes related to immune function and inflammation were upregulated in both collections. In addition, a co-expression module related to immune/inflammation response that was generated by combining mRNA-Seq data from both collections was significantly associated with disease status. The immune/inflammation-related co-expression module was positively correlated with levels of C-reactive protein (CRP), cortisol and several immune modulator proteins in the serum of the same individuals and was also positively correlated with CRP, cortisol and pro-inflammatory cytokines in the frontal cortex of the same individuals. In addition, we found a substantial number of nodes (genes) that were common to our schizophrenia-associated immune/inflammation module from the pooled data and a module we generated from lippopolysaccharides-treated mouse model data. These results suggest that the CP of individuals with schizophrenia are responding to signals from the periphery by upregulating immune/inflammation-related genes to protect the brain and maintain the homeostasis but nevertheless fails to completely prevent immune/inflammation related changes in the brain.

Translational Psychiatry (2016) 6, e964; doi:10.1038/tp.2016.229; published online 29 November 2016

\section{INTRODUCTION}

Schizophrenia is a common psychiatric disorder that is believed to be caused by a complex interaction between multiple genetic and environmental factors. Although the pathophysiology of the disorder is not well understood, there are now numerous studies that show abnormalities in markers of the immune and inflammatory systems in the blood ${ }^{1-6}$ and cerebrospinal fluid $(\mathrm{CSF})^{7-9}$ of individuals with schizophrenia. There are also a number of studies showing abnormalities in markers of immune function and inflammation within the brains of schizophrenia cases, ${ }^{10-16}$ however, there is scant evidence for lymphocytic infiltration into the brain, ${ }^{17}$ and there is contradictory and inconclusive evidence for activation of the microglia or resident immune cells of the brain in schizophrenia cases. ${ }^{14,17,18}$ In contrast, our recent transcriptome sequencing data from the hippocampus ${ }^{15}$ indicated that the abnormally expressed molecules related to immune and inflammation pathways were more likely to be expressed in endothelial cells of blood vessels, in blood monocytes within the blood vessels and in the perivascular astrocytes in the schizophrenia cases, than in lymphocytes or microglia. Thus, the blood-brain-barrier (BBB) may be functioning abnormally in the schizophrenia cases or cells proximal to the BBB may be responding to aberrant factors in the blood of patients with schizophrenia that then causes abnormal function in neurons. Previous evidence for abnormalities in the BBB was provided by a laser capture microdissection study of cerebral vascular endothelial cells that found abnormal expression of genes relating to the inflammatory process in schizophrenia. ${ }^{19}$ An electron microscope study also found ultrastructural abnormalities in endothelial cells and astrocytic end-feet in the frontal cortex of patients with schizophrenia. $^{20}$

In addition to the endothelial BBB, there is also the epithelial blood-cerebrospinal fluid-barrier (BCSFB), which together, protects the brain from invading microorganisms and maintain the homeostatic environment of the central nervous system (CNS). Whereas, the $B B B$ is defined by the cerebral vasculature, the choroid plexus (CP) defines the BCSFB. The CP is a very specialized, highly vascular ependymal structure in the ventricles of the brain where CSF is produced. The CP is structured to restrict cellular and molecular traffic between the blood and $\mathrm{CSF}^{21}$ and acts as a filtration system, removing metabolic waste, foreign substances and excess neurotransmitters from the CSF. An inflammatory response is induced in the $\mathrm{CP}$ after injection of a peripheral inflammatory stimulus such as the bacterial endotoxin lipopolysaccharide (LPS) ${ }^{22}$ or a parasite. ${ }^{23}$ This response may be expected given the tropism of various bacteria, parasites and viruses for the CP. ${ }^{23-25}$ Several studies have also shown changes in CP gene regulation after chronic stress ${ }^{26}$ and in response to treatment ${ }^{27}$ indicating that the $\mathrm{CP}$ may be an important site to monitor CNS response to environmental stimuli. Given the unique structural and functional position of the $\mathrm{CP}$ at the interface between two circulating fluid compartments, its ability to respond to environmental stimuli and its ability to influence the brain via immune and humoral signals, we sequenced the CP transcriptome of 29

\footnotetext{
${ }^{1}$ Stanley Brain Research Laboratory, Stanley Medical Research Institute, Rockville, MD, USA and 2Department of Bio and Brain Engineering, KAIST, Daejeon, Korea. Correspondence: Dr D Lee, Department of Bio and Brain Engineering, KAIST, 291 Daehak-ro, Yuseong-gu, Daejeon 305-701, Korea or Dr MJ Webster, Stanley Brain Research Laboratory, Stanley Medical Research Institute, 9800 Medical Center Drive, Suite C-050, Rockville, MD 20850, USA.

E-mail: dhlee@kaist.ac.kr or websterm@stanleyresearch.org

Received 13 November 2015; revised 19 September 2015; accepted 28 September 2016
} 
subjects with schizophrenia and 26 unaffected controls from the Stanley Array Collection to further understand the molecular pathways that may be impacting the CNS of individuals with schizophrenia. As a replication study, we sequenced the CP transcriptome of 19 subjects with schizophrenia and 19 unaffected controls from the New Stanley Collection.

\section{MATERIALS AND METHODS}

\section{CP samples}

RNA for the sequencing study was extracted from post-mortem $\mathrm{CP}$ at the level of the posterior lateral ventricle from 30 subjects with schizophrenia and 26 unaffected controls that belong to the Stanley Array Collection (AC). It should be noted that one schizophrenia case that died from septicemia and acute pancreatitis was excluded from the downstream analysis because this factor could have directly contributed to elevated immune markers at time of death. The two groups were previously matched for age, gender, race, post-mortem interval (PMI), mRNA quality, brain $\mathrm{pH}$ and hemisphere. The subjects in this study have been included in numerous neuropathology studies, gene expression microarray studies and single-nucleotide polymorphism association studies. The data from these studies is available at http://sncid.stanleyresearch.org (SNCID). A replication sequencing study was conducted on 20 subjects with schizophrenia and 19 unaffected controls that belong to the New Stanley Collection. One schizophrenia case was excluded from downstream analysis because the patient had an autoimmune condition resulting in alopecia. Descriptive variables for samples from both tissue collections are listed in Supplementary Table 1. To compare continuous variables such as age, brain $\mathrm{pH}, \mathrm{PMI}$ and RNA integrity number (RIN) between schizophrenia and controls we first performed a Shapiro-Wilk test for normality and found brain $\mathrm{pH}$ and PMI of the AC samples as well as PMI and RIN of the New Stanley Collection samples were not normally distributed (all $P<0.05$ ). Therefore, the nonparametric Kruskal-Wallis test was performed for group differences of continuous variables and Fisher's exact test performed for nominal variable.

\section{Library preparation and mRNA sequencing}

Extracted total RNA was analyzed using an Agilent 2100 Bioanalyzer (Agilent, Palo Alto, CA, USA). Total RNA ( $1 \mu \mathrm{g})$ was subjected to two rounds of hybridization to oligo(dT) beads (Dynal, Waltham, MA, USA). The resulting mRNA was then used as a template for CDNA synthesis. The mRNA was randomly fragmented to between 200 and 700 bp by focused acoustic shearing (Covaris, Woburn, MA, USA) and converted to first strand cDNA using Superscript III (Invitrogen, Carlsbad, CA, USA), followed by second-strand cDNA synthesis using Escherichia coli DNA pol I (Invitrogen). The double stranded cDNA library was further processed by Illumina Genomic DNA Sample Prep kit (Illumina, San Diego, CA, USA). It involved end repair using T4 DNA polymerase, Klenow DNA polymerase and T4 polynucleotide kinase followed by a single $\langle\mathrm{A}\rangle$ base addition using Klenow $3^{\prime}$ to $5^{\prime}$ exo- polymerase, and was ligated with Illumina's adaptor oligo mix using T4 DNA ligase. Adaptor-ligated library was size selected by electrophoretic separation on a $2 \%$ agarose gel and excising of the library smear at $500 \mathrm{bp}$. The library was PCR amplified for 18 cycles using Phusion polymerase and purified by Qiaquick PCR Purification Kit (Qiagen, Valencia, CA, USA) and quantified by Quant-iT picogreen dsDNA Assay Kit (Invitrogen) following the manufacturer's protocol. We then prepared HiSeq 2000 paired-end flow cell on the supplied Illumina cBot and generated 101- bp paired-end sequence reads on the Illumina HiSeq 2000 platform following the manufacturer's protocol. Images taken during the sequencing reactions were processed in five stages by Illumina's software: Image analysis is performed by the instrument control software (Hiseq Control Software, Illumina), base calling is performed by the instrument control software's Real Time Analysis (RTA).

\section{Reads mapping and differentially expressed genes}

All reads were mapped to UCSC H. sapiens reference genome (build hg19) using TopHat v2.0.9 with UCSC refFlat gene model annotation file on the $G$ parameter. ${ }^{28}$ We used the expected mean inner distance between mate paired-ends as $-r$ parameter. TopHat calls Bowtie v2.1.0 to perform the alignment with no more than two mismatches. We used the pre-built index files of UCSC $H$. sapiens hg19, which are downloaded from the TopHat homepage (http://tophat.cbcb.umd.edu/index.html). The quantification of gene expression was accomplished by HTseq v0.5.3p9 (ref. 29) and edgeR package. ${ }^{30}$ All mapped read counts of the genes were counted by htseq-count (subprogram of HTseq) ${ }^{29}$ with UCSC refFlat gene model annotation file, no strand specific option, and intersectionnonempty option. The differentially expressed genes between schizophrenia and controls were identified using edge $\mathrm{R}^{30}$ and the false discovery rates $<0.05$ were considered significant.

\section{Mouse CP gene expression data}

Microarray data from the $\mathrm{CP}$ of a previous mouse study ${ }^{31}$ was downloaded from the GEO database. The datasets (GSE23714) consist of normalized gene expression data from Illumina Whole-genome Mouseref-8 expression beadchips (Illumina). The data was from the CP of mice injected with LPS and killed 1, 3 and $72 \mathrm{~h}$ after the treatment. Control mice were injected with saline solution $(n=10){ }^{31}$ The top 5000 probes from each treatment condition were filtered based on the coefficient of variation to select genes showing a high dynamic range of gene expression ${ }^{32,33}$ and were then used as input for weighted correlation network analysis (WGCNA) $)^{34}$ to generate a network.

\section{Gene co-expression network analysis}

We constructed co-expression networks using RNA-Seq data for all genes expressed in the $\mathrm{CP}$ and pooled the data from both schizophrenia and control groups. Surrogate variable analysis (SVA) ${ }^{35}$ was used to identify the potential confounding effects in the RNA-Seq data and then the confounding effects were adjusted using the resulting surrogate variables from the SVA. The standardized residuals from the linear regression were used to generate gene co-expression networks using WGCNA. ${ }^{34}$ To construct a weighted co-expression network we selected the power for which scale-free topology fitting index $\left(R^{2}\right)$ is $\geqslant 0.9 .^{36}$ Correlation analyses were performed between co-expression modules and traits such as diagnosis and demographic or clinical variables to identify modules that were associated with disorder and/or confounding factors. Multivariate permutation testing of correlations was performed to adjust for multiple testing using MPTCorr.r package ${ }^{37}$ (http://www.psych.umn.edu/faculty/ waller/downloads/mpt/mptcorr.r). The statistical package was developed to perform multivariate permutation tests of the correlations between a single criterion variable and multiple predictor variables. ${ }^{37}$ We used a trait as a criterion variable and the eigengene values in all modules as multiple predictor variables. Adjusted $P$-values $<0.05$ were considered significant. The network connections were visualized using VisANT. ${ }^{38}$

Network comparisons between schizophrenia and controls Separate co-expression networks were generated for each group independently by using only schizophrenia data or only control data in the network. We could then compare the co-expression modules of the schizophrenia group to that of the unaffected control group. Module preservation was examined using a permutation test procedure implemented in the WGCNA. ${ }^{39}$ Summary preservation $Z$ score $\left(Z_{\text {summary }}\right)$ was used to determine whether a co-expression module was preserved in a test network as compare with a reference network. Previous threshold guidelines were followed: if $Z_{\text {summary }}<2$, then there is no evidence that the module is preserved. If $2<Z_{\text {summary }}<10$, then there is weak to moderate evidence that the module is preserved. If $Z_{\text {summary }}>10$, then there is strong evidence that the module is preserved. ${ }^{39}$

\section{Consensus module analysis}

Co-expression modules from two independent datasets, the $A C$ and the New Stanley Collection, were compared to identify the consensus modules. $P$-values of significance for each of the pairwise overlaps were obtained by permutation tests in $R$. As we performed 1 million permutations, the lowest possible $P$-value we can obtain from our analysis is $1 e-05$. The immune-related module from the combined data of the AC and the New Stanley Collection and the immune-related module from the mouse $\mathrm{CP}$ gene expression data were compared by the permutation test. The consensus network connections were identified and visualized using Cytoscape. ${ }^{40}$ 
Correlation analysis between the immune/inflammation related co-expression module and markers in the serum and frontal cortex of the same cases

Correlation analyses were performed between the immune/inflammation related co-expression module and protein markers related to the same biological processes. The protein levels were measured in serum and frontal cortex of the same individuals from the $A C$ using the DiscoveryMAP multiplexed immunoassay panel at Myriad-RBM (Austin, TX, USA). ${ }^{41}$ The raw data is available in the SNCID. We downloaded the raw data and used it as traits for the correlation analysis using WGCNA. $P$-values $<0.05$ were considered significant.

Functional annotation

DAVID (http://david.abcc.ncifcrf.gov/home.jsp) was used to identify the biological processes that were significantly over-represented by differentially expressed genes between schizophrenia and normal controls as well as genes included in the co-expression modules. ${ }^{42}$ Gorilla (http://cblgorilla.cs.technion.ac.il/) was used to annotate significantly enriched biological processes in the top 5000 probes that were selected from data of mice $3 \mathrm{~h}$ post LPS treatment and controls, ${ }^{43}$ because DAVID does not allow more than 3000 genes or probes as input. $P$-values $<0.05$ were considered significant.

Code availability

Codes used for permutation tests are available from the corresponding author.

\section{RESULTS}

Differential gene expression in CP of individuals with schizophrenia

We analyzed RNA-Seq data from CP of 29 subjects with schizophrenia and 26 unaffected controls from the array collection. There were no significant differences of age, sex, PMI, RIN and brain $\mathrm{pH}$ between schizophrenia and controls (Supplementary Table 1). For each sample, we generated an average of 51 million 101-bp reads (Supplementary Table 2). Sequence reads were mapped to the human reference genome (hg19) and for each sample the average number of mapped reads was 36.6 million. We then quantified the aligned read counts and performed statistical analysis. Twenty-seven genes were significantly differentially expressed between schizophrenia and unaffected controls in the $\mathrm{CP}$ at false discovery rate $<0.05$ (Supplementary Table 3). The expression of 23 genes was upregulated, and 4 genes were downregulated.

Although 25 biological processes were significantly enriched in the upregulated genes in the $\mathrm{CP}$ of schizophrenia patients (Supplementary Table 4), there was no significantly enriched biological processes in the down-regulated genes. Defense, immune and inflammatory responses and amino acid transport were the main biological processes that were significantly enriched in the differentially expressed genes (Supplementary Table 4). The genes involved in the defense/inflammatory/ immune responses were predominantly upregulated in the CP of schizophrenic patients as compared with the unaffected controls. Although the functional annotation tool listed nine genes, IL18R1, SLC11A1, SERPINA3, PLA2G2A, PTX3, THBS1, CD163, TNFSF14 and CLEC4D, as defense/inflammatory/immune responserelated, our independent interrogation of the other differentially expressed genes revealed that two additional genes are also involved in immune response, for example, LRG1 (leucine-rich alpha-2-glycoprotein 1) is involved in granulocyte differentiation ${ }^{44}$ and STEAP4 (STEAP family member 4) is a metalloreductase involved in the inflammatory response. ${ }^{4,46}$

Gene co-expression network analysis

An unsupervised co-expression network analyses was performed by combining the RNA-Seq data, which was adjusted for covariates using SVA from all schizophrenia cases and controls to identify the co-expression modules associated with schizophrenia. Of the 18 co-expression modules that were generated

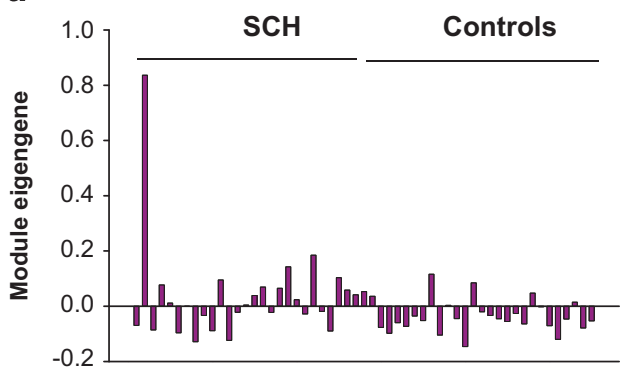

b

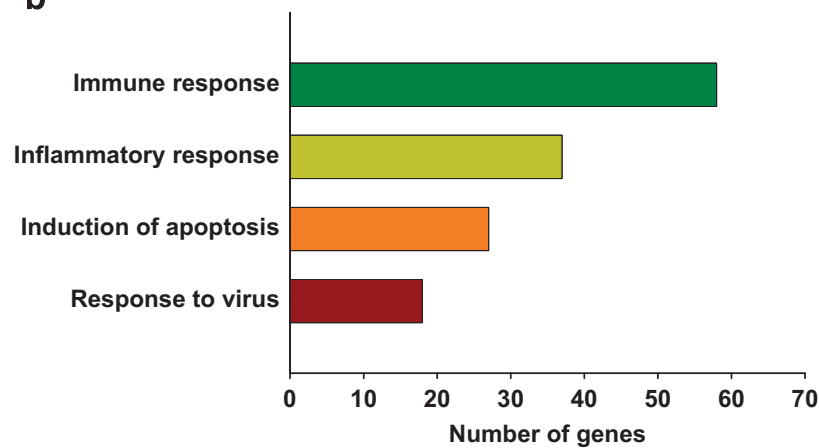

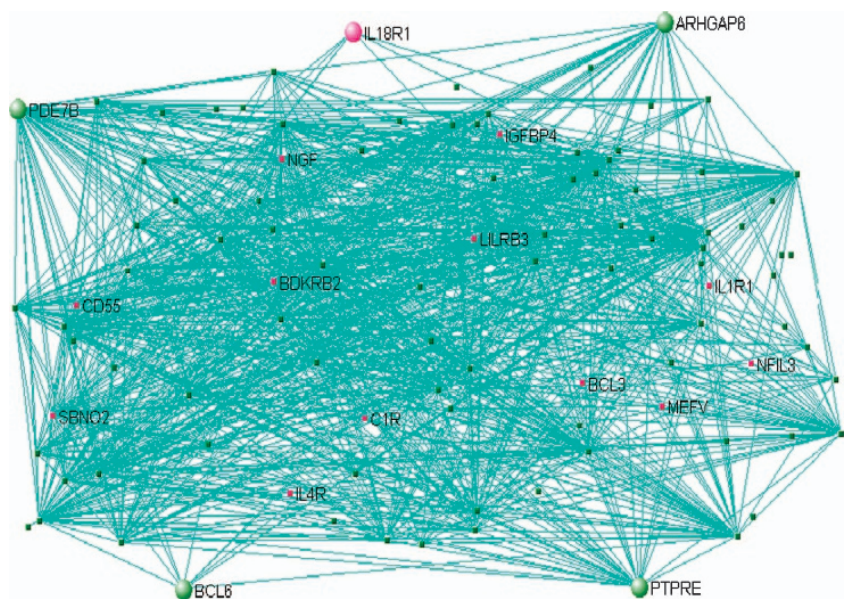

.


Table 1. Correlation between the co-expression module (S_M16) associated with the immune/inflammation response in the choroid plexus and protein markers in serum and frontal cortex of the same individuals

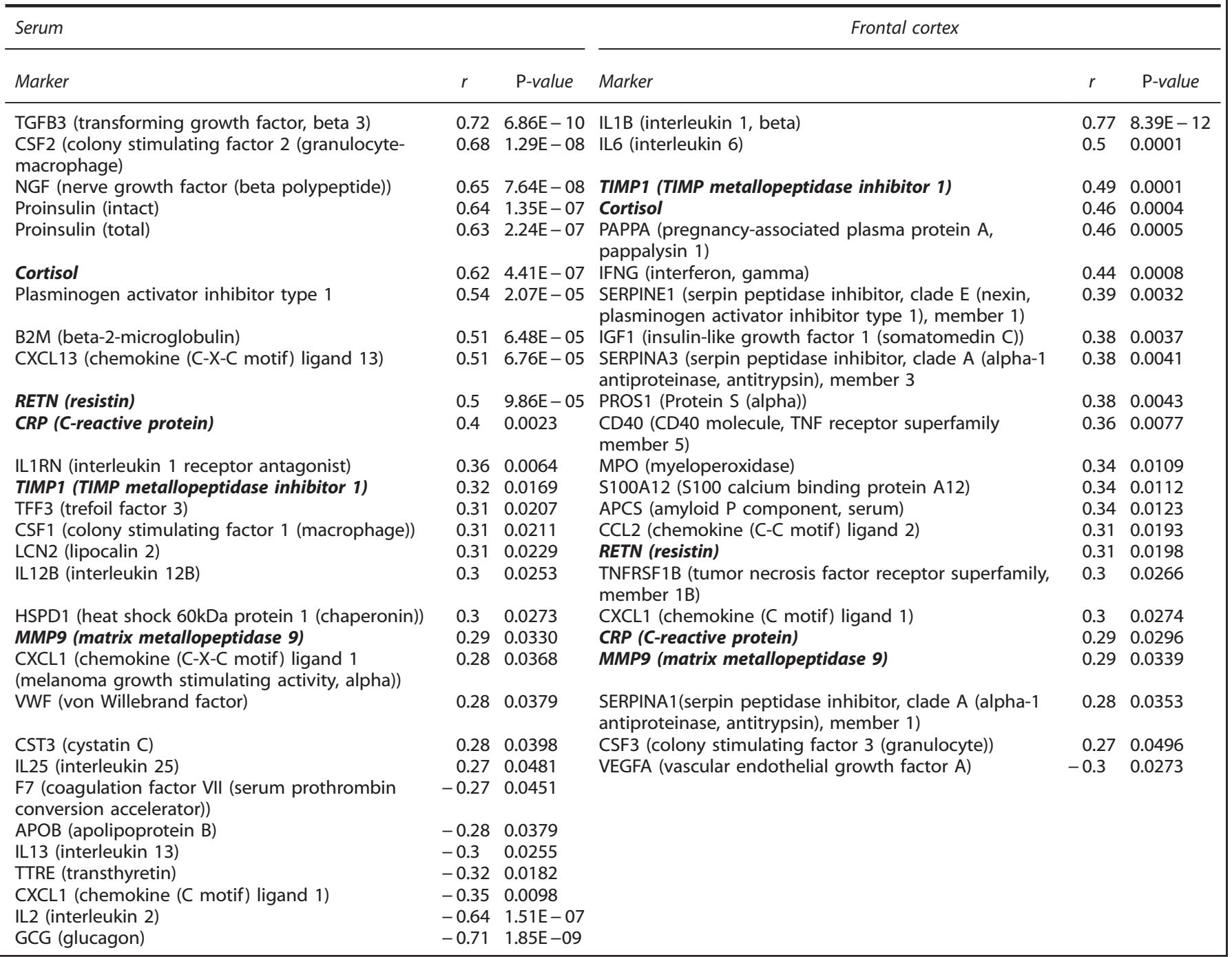

with this combined data, none of the modules were significantly associated with schizophrenia using the multivariate permutation testing of correlations (Supplementary Table 5). Among all modules, the S_M16 module was the most significantly associated with schizophrenia, however, the $P$-value did not reach statistical significant level $\left(r=0.3, P_{\text {adj }}=0.26\right)$. The S_M16 was a large module containing 629 genes. The eigengene values in this module were over expressed in schizophrenia cases as compared with controls (Figure 1a), and immune and inflammation response, apoptosis, response to virus and hypoxia were significantly enriched in this module (Figure 1b; Supplementary Table 6). IL18R1 (interleukin 18 receptor 1), PTPRE (protein tyrosine phosphatase, receptor type E), ARHGAP6 (Rho GTPase activating protein 6), PDE7B (phosphodiesterase $7 \mathrm{~B}$ ) and BCL6 (B-cell CLL/lymphoma 6) are the top 5 hub genes in this module (Figure 1C).

Comparing the separate co-expression networks of schizophrenia and unaffected controls

To further explore fundamental differences in gene-gene interactions in the $\mathrm{CP}$ of individuals with schizophrenia as compared with controls, we constructed co-expression networks for schizophrenia and controls separately. We then compared the coexpression modules for schizophrenia to those for controls, or vice versa and found that almost all the modules were conserved between the schizophrenia cases and controls indicating that there is no difference in the gene-gene interactions between cases and controls. However, two modules were only weakly conserved indicating that there are some possible differences in the gene-gene interactions between the cases and controls (Supplementary Table 7). One module, C_only1_M8 was only weakly conserved in schizophrenia as compared with controls and therefore more likely to be specific to controls, whereas the other module, SCH_only_M7 was only weakly conserved in controls as compared with schizophrenia $\left(2<Z_{\text {summary }}<10\right.$, Supplementary Table 7) and therefore more likely to be schizophrenia specific. The control-specific module, C_only1_M8, was negatively correlated with brain $\mathrm{pH}$ (Supplementary Table 8). Regulation of signal transduction, cell cycle and transcription were the main biological processes associated with this module (Supplementary Table 9). The schizophrenia specific module, SCH_only_M7, was not correlated with any covariates we tested (Supplementary Table 10) and contained 76 genes with BAG3, DNAJB1,DNAJB4, HSPAH and HSPA6 the top 5 hub genes in this module (Supplementary Figure 1a). Response to organic substances, regulation of apoptosis and cell proliferation were the process significantly enriched in the co-expressed genes (Supplementary Figure 1b; Supplementary Table 11). 


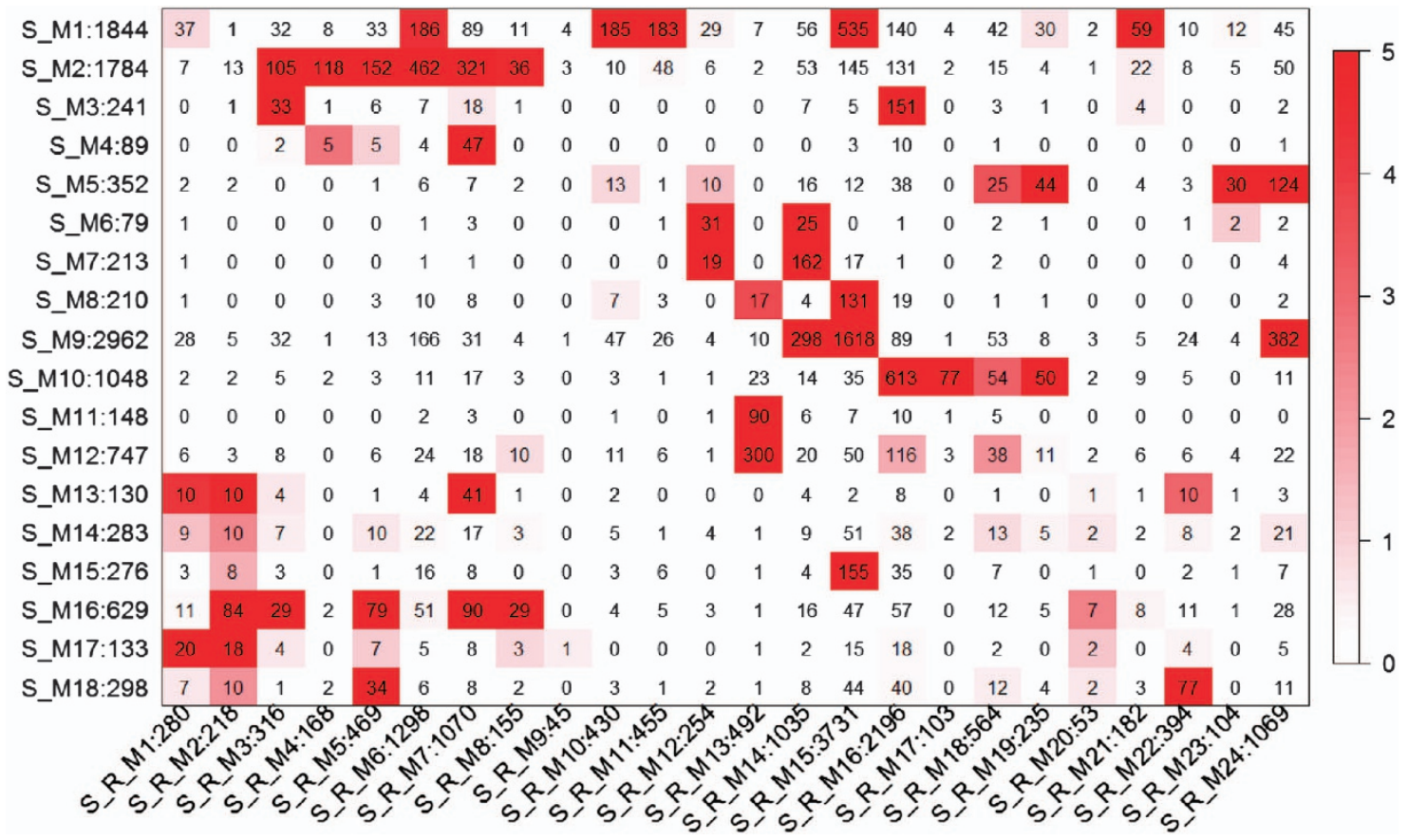

Figure 2. Pairwise comparisons of the modules between the AC data and the New Stanley data. The color code of the heatmap encodes - log $(P$-value). The $P$-values were calculated by permutation test for the overlap of the two modules. The numbers in the heatmap indicate gene counts in the intersection of two modules. The label to the left of the $y$-axis of a heat map indicates modules of the AC data and gene counts in the modules. The label to the bottom of the $x$-axis of the heatmap indicates modules of the New Stanley Collection data and gene counts in the modules. AC, Array Collection.

Association between the immune/inflammation related coexpression module and markers in the serum and frontal cortex

A correlation analysis was performed to explore the association between the S_M16 module and the protein markers measured in the serum and frontal cortex of the same individuals. Thirty and twenty-three markers related to immune/inflammation response were significantly correlated with the $\mathrm{S} \_\mathrm{M} 16$ module in the serum and the frontal cortex, respectively (Table 1). Five markers, including CRP, cortisol, TIMP metallopeptidase inhibitor 1 (TIMP1), matrix metallopeptidase 9 (MMP9) and resistin (RETN) were positively correlated with the module in both the serum and the frontal cortex. Immune modulators such as IL1RN and IL25 were positively correlated with the module in the serum, whereas proinflammatory cytokines such as IL1B and IL6 were positively correlated with the module in the frontal cortex.

Validation of RNA-seq results using an independent tissue collection

We used an independent tissue collection to replicate the differentially expressed genes and the co-expression modules associated with schizophrenia in the $\mathrm{CP}$. There were no significant differences of age, brain $\mathrm{pH}, \mathrm{RIN}$ and PMI between schizophrenia and controls in this cohort (Supplementary Table 1). However, sex was significantly different between schizophrenia and controls in this cohort ( $P=0.001$; Supplementary Table 1$)$. We generated an average of 99.9 million 101-bp reads and for each sample the average number of mapped reads was 90.9 million (Supplementary Table 12). Although there were 33 genes significantly differentially expressed between schizophrenia and unaffected controls in the CP at false discovery rate $<0.05$ (Supplementary Table 13), there was no significantly enriched biological processes in the genes. However, there was a significant overlap between the differentially expressed genes from the $\mathrm{AC}$ and the New Stanley Collection data $(P<1 \mathrm{e}-05)$. Three genes, SERPINA3, ADAMTS17 and CISH were differentially expressed in both collections.
Twenty-four co-expression modules were built using the combined data from schizophrenia and controls and although one (S_R_M9) was significantly associated with schizophrenia, it was also highly significantly correlated with sex (Supplementary Table 14), and therefore excluded from downstream analysis. When comparing the nodes (genes) in the modules from the New Collection to those from the AC data, we found significant overlap of genes common to both datasets (Figure 2). Although most modules from the New Stanley Collection data have consensus with modules from the AC network (Figure 2), two modules (S_R_M9 and S_R_M20) did not have any consensus with AC modules. The immune-related module, S_M16 from the AC data had significant overlap of common nodes (genes) with five coexpression modules from the New Stanley Collection, S_R M2, S_R_M3，S_R_M2，S_R_M5，S_R_M7 and S_R_M8. Moreover, S_M16 has the most significant overlap with the S_R_M2, where 84 nodes (38\%) were also included in the S_M16 module $(P<1 \mathrm{e}-05)$ (Figure 2). The module S_R_M2 contained 218 genes and cytokine-mediated signaling pathway, defense/ inflammatory responses and apoptosis were significantly enriched in this module (Supplementary Table 15).

Next, we built co-expression networks for schizophrenia and controls separately and then compared them to each other in an attempt to replicate the results from the AC samples. One module, C_R_only_M10, was weakly conserved in schizophrenia as compared to controls and therefore likely to be control specific. (Supplementary Table 16). The module was also significantly correlated with PMI (Supplementary Table 17) and only contained 30 genes that were enriched for gaseous exchange, regulation of cell migration and cell proliferation (Supplementary Table 18).

Of the 23 co-expression modules generated from the schizophrenia only data, two were not preserved at all (S_R_only_M12 and M18) and 3 (S_R_only_M1, M7 and M20) were only weakly preserved in controls and therefore all are likely to be specific to schizophrenia (Supplementary Table 16). The five modules were not significantly correlated with any confounding variables tested 
(Supplementary Table 19). The S_R_only_M1 module contained 106 genes enriched for immune response and response to virus (Supplementary Table 20). The S_R_only_M12 module was not preserved at all in controls and contained 38 genes enriched for negative regulation of cell proliferation and apoptotic mitochondrial change (Supplementary Table 21). The S_R_only_M18 module was also not preserved at all in controls and contained 116 genes enriched for response to nutrients and regulation of retinoic acid receptor signaling pathway (Supplementary Table 22). The remaining two modules, which were only weakly preserved in controls, contained genes significantly enriched for ion homeostasis and neuropeptide signaling pathways (Supplementary Table 23).

Network analysis of the pooled data from both AC and new stanley collection

The co-expression module from the $A C$ related to immune/ inflammation response was successfully replicated in the independent RNA-seq data set. Although both of these modules were also the modules most significantly associated with schizophrenia, the $P$-values did not reach a statistical significant level in multivariate permutation testing of correlations. Because the sample size was relatively small for each analysis, we repeated the analysis by combining the two datasets to increase the sample size and to increase detection power.

We generated 23 co-expression modules with the combined data. One module (S_Co_M16) was significantly associated with schizophrenia $\left(r=0.311, P_{\text {adj }}=0.03\right.$; Supplementary Table 25$)$. The eigengene values in this module were over expressed in schizophrenia cases as compared with controls (Figure 3a), and immune and inflammation response, regulation of apoptosis and response to virus were significantly enriched in this module (Figure 3b; Supplementary Table 26). IL18R1 (interleukin 18 receptor 1), IL4R (interleukin 4 receptor), IGFBP4 (insulin-like growth factor binding protein 4), CASP4 (caspase 4) and BCL6 (B- cell CLL/lymphoma 6) are the top 5 hub genes in this module (Figure 3c).

Comparison of modules from our human CP study and those from a mouse model

To explore the possible causes of the activated immune/ inflammation modules in the CP of the schizophrenia cases, we compared the immune/inflammation module from the combined data to a co-expression module from a well-validated mouse model. ${ }^{31}$ First, we generated co-expression networks from data collected from the CP of LPS-treated mice at 1, 3 or $72 \mathrm{~h}$ post treatment and combined the data with that from the control mice. To generate a network using genes showing a high dynamic range of gene expression, the top 5000 probes were filtered based on the coefficient of variation. A total of 15 and 16 co-expression modules were generated with data from mice 1 and $72 \mathrm{~h}$ post LPS treatment and controls, respectively. None of the modules was correlated with LPS treatment. In contrast, the co-expression modules generated with data from mice $3 \mathrm{~h}$ post LPS treatment and controls did produce a module correlated with LPS treatment. The functional annotation of the top 5000 probes from the $3 \mathrm{~h}$ post LPS treatment indicated that immune system process and immune/inflammation response were highly enriched in the list (Supplementary Table 27). Of the 15 co-expression modules built, one module, LPS_3h_M2, was significantly positively associated with LPS treatment $\left(r=0.99, P_{\mathrm{adj}}<0.001\right)$. Immune and inflammation response, regulation of gene expression and response to LPS were significantly enriched in this module (Supplementary Table 28). These results indicate that the most robust acute response occurred at $3 \mathrm{~h}$ post LPS injection, which is consistent with the results from the original study. ${ }^{31}$

We then compared the nodes (genes) in this module to those in the schizophrenia-associated module, S_Co_M16 and found a significant number of nodes (62) that were common to both LPS_3h_M2 and the schizophrenia-associated module $(P<1 \mathrm{e}-$ 05; Figure 4a). Immune and inflammation response, regulation of a

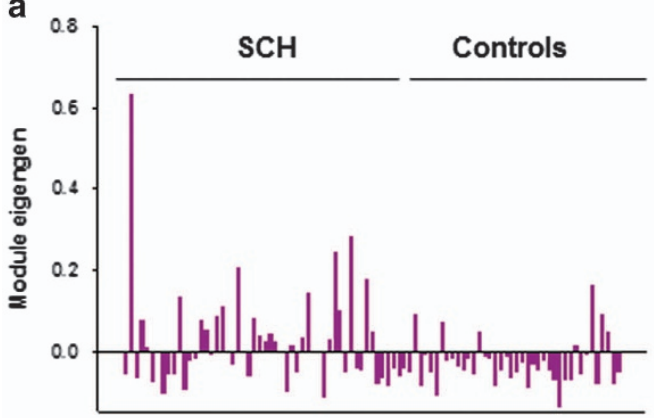

b

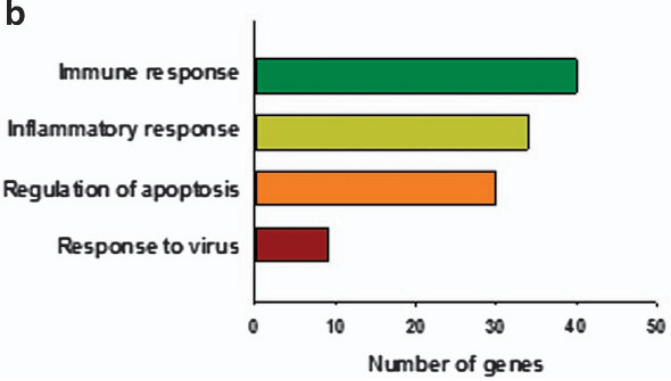

C

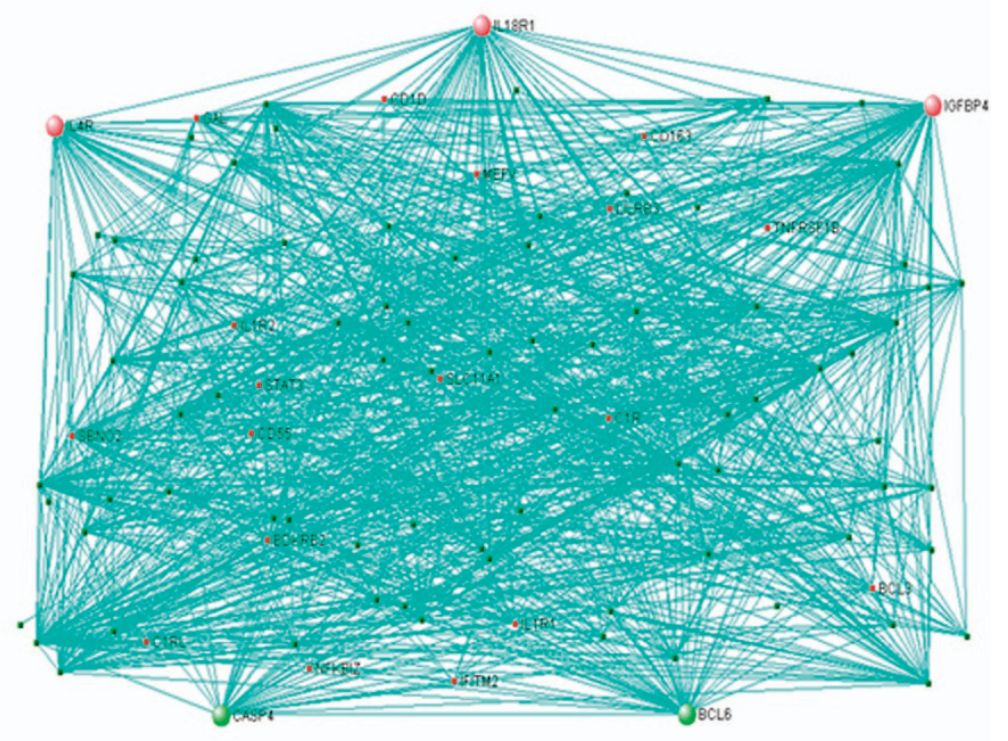

Figure 3. Co-expression module of the combined data associated with schizophrenia and related to immune/inflammation response in choroid plexus. The eigengene values across samples in the S_Co_M16 module (a); major biological processes (gene ontology) significantly enriched in the genes in the co-expression module (b) and visualization of the module (c). The network connections of top 100 genes with topological overlap above the threshold of 0.05 were visualized using VisANT. ${ }^{38}$ The hub genes are larger circles in the network. Genes related to immune/inflammation responses are red. 
a

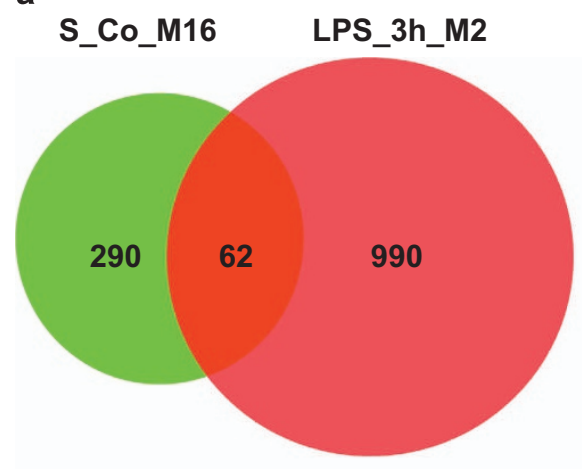

b

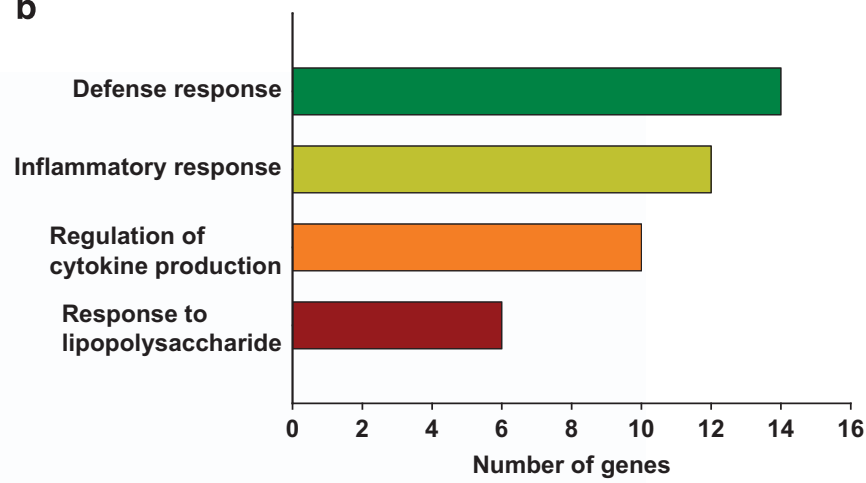

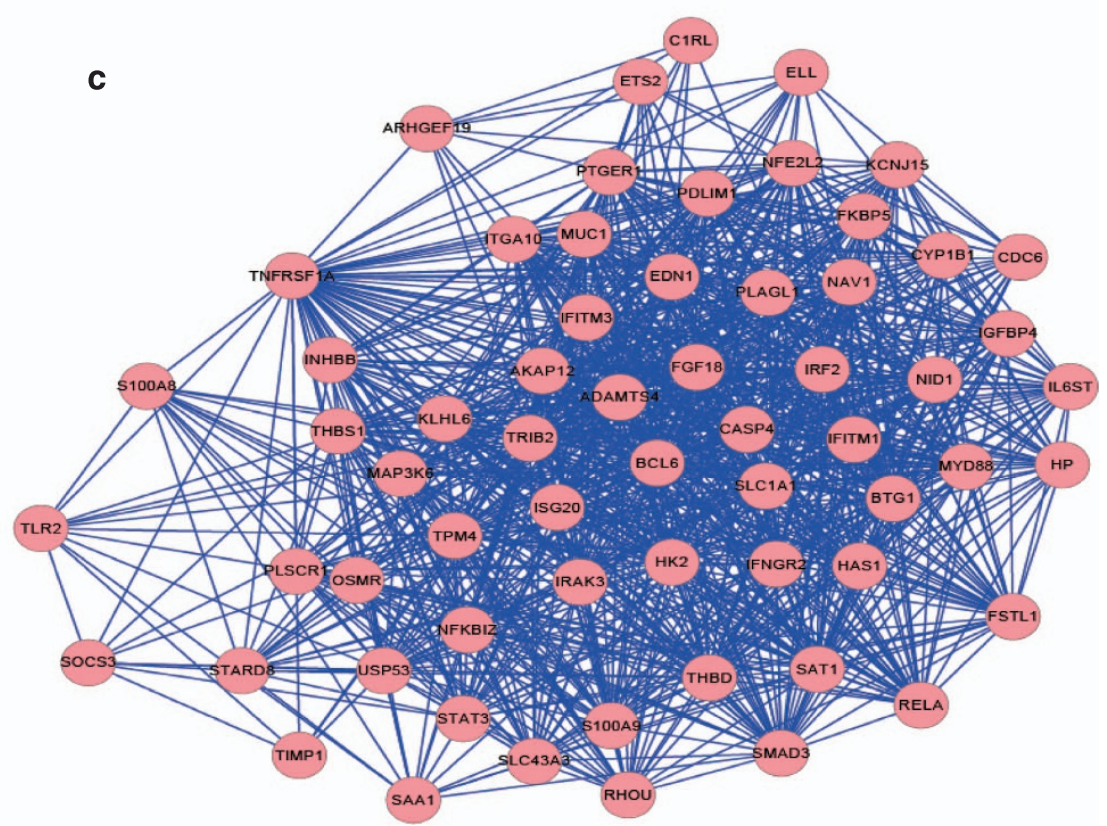

Figure 4. Comparative network analysis of the immune/inflammation related module, S_Co_M16, from the choroid plexus of schizophrenia and controls compared with mice $3 \mathrm{~h}$ post lippopolysaccharide treatment. Venn diagram shows the number of common and unique genes between the schizophrenia-associated module S_Co_M16 and the mouse LPS_3h_M2 module (a). Major biological processes (gene ontology) significantly enriched in the genes common to both co-expression modules (b) and visualization of the network connections of the common genes between the two modules using Cytoscape (c). ${ }^{40}$

cytokine production and response to LPS were significantly enriched in the common genes (Supplementary Table 29; Figures $4 b$ and $c)$.

\section{DISCUSSION}

The CP not only produces CSF, but is also a physical and 'biochemical' barrier that maintains the internal microenvironment of the CNS. ${ }^{47}$ To maintain this homeostasis the CP is modulated by, and must respond to peripheral inflammatory signals. The hypothesis that neuroimmune mechanisms are having a role in the eitiology and pathophysiology of psychotic disorders is generating renewed interest. ${ }^{48}$ Consequently considerable evidence is accumulating from both the periphery ${ }^{3}$ and brain $^{10-15,49,50,51}$ to support this hypothesis, and networks constructed from candidate genes for schizophrenia and carcinoma show that immune-related genes are associated with both schizophrenia and tumor suppression. ${ }^{52}$ Few studies have investigated the $\mathrm{BBB}^{19,20}$ or BCSFB/CP ${ }^{53,54}$ in schizophrenia. Here, we investigate the genes and related biological processes that are dysregulated in the $\mathrm{CP}$ of individuals with schizophrenia as compared with unaffected controls. Genome-wide expression profiles of the $\mathrm{CP}$, comparing the schizophrenia group to the matched controls, were generated from two independent tissue collections. We built co-expression networks from the combined gene expression data of both tissue collections and found a module related to immune/inflammation response over expressed in schizophrenia as compared with unaffected controls in both tissue collections. In addition, we found a schizophrenia specific co-expression module related to apoptosis in both tissue collections.

The majority of differentially expressed genes were upregulated in schizophrenia in both collections and many of the genes are involved in immune function and inflammation including the three genes common to both collections, SERPINA3, CISH and ADAMTS17. SERPINA3 (Serpin Peptidase Inhibitor, Clade A (Alpha-1 Antiproteinase, Antitrypsin), Member 3) also known as alpha-1antichymotrypsin, is a serine protease inhibitor and an acute phase protein that is induced during inflammation. However, SERPINA3 inhibits the activity of proteases, such as cathepsin G thereby limiting inflammation, inhibiting apoptosis and protecting tissues. ${ }^{55}$ Endothelial and epithelial cells, macrophages and astrocytes express SERPINA3. Numerous studies of prefrontal cortex have consistently found SERPINA3 mRNA expression to be upregulated in schizophrenia ${ }^{10,11,14,16,56}$ and here we also find it upregulated in the CP of two independent collections. Moreover, 
alpha-1-antichymotrypsin protein levels are elevated in the frontal cortex and serum of these same cases (SNCID) and the immune/ inflammation related co-expression module, S_M16 was positively correlated with alpha-1-antichymotrypsin protein levels in the frontal cortex of the same individuals (Table 1). Thus, it appears that the widespread and consistent upregulation of SERPINA3 mRNA and protein in schizophrenia may be an attempt to maintain the homeostasis of the CNS in response to a peripheral stimulus. Likewise, $\mathrm{CISH}$ (Cytokine Inducible SH2-Containing Protein) inhibits IL-2 and IL-3 signaling and through a feedback mechanism has an important role in controlling the magnitude and duration of the cellular response to extracellular stimuli. ${ }^{57}$ Polymorphisms in the CISH gene are associated with susceptibility to various infectious pathogens. ${ }^{58}$ Finally, ADAMTS17 (ADAM metallopeptidase with thrombospondin type 1 motif, 17) is a zincdependent protease and although the precise function of ADAMTS17 is not yet known, the general function of such enzymes in normal tissue remodeling and wound healing may be relevant to maintaining the integrity of the $C P$ as it responds to external stimuli. Together, the upregulation of these three genes in both cohorts indicates that the $\mathrm{CP}$ may be mounting a protective stance in response to a peripheral stimulus in the schizophrenia patients.

Both tissue collections each produced a co-expression module, S_M16 and S_R_M2 that were related to immune/inflammation response. The $P$-values for significance of associations between the modules and schizophrenia were the lowest among modules, but did not reach a statistical significant level after adjusting for multiple testing. None of the modules generated from either RNASeq data set were significantly associated with schizophrenia, indicating that the sample size may not have been adequate for the network analysis. Therefore, we performed a network analysis with the pooled data of the two tissue collections and identified an immune-related module, S_Co_M16 that was significantly associated with schizophrenia. All three modules associated with schizophrenia were also related to immune/ inflammation response and contained four interleukin receptor genes, ILIR1, IL4R, IL15RA and IL18R1. The genes IL1R1 and IL18R1 are receptors for IL1 and IL18, which are both pro-inflammatory cytokines that induce and maintain the inflammatory cascade. ${ }^{59,60}$ Studies of IL1B serum levels have found consistent abnormalities in schizophrenia $^{3}$ and the co-expression module, S_M16 was positively correlated with IL1B protein levels in the frontal cortex of these same individuals (Table 1). Similarly IL18 serum levels are consistently upregulated in patients with schizophrenia ${ }^{61-65}$ and IL18 protein levels are significantly increased in the frontal cortex of subjects with schizophrenia (SNCID). IL18 serum levels are also associated with hippocampal volume in schizophrenia ${ }^{65}$ and peripheral inflammatory insult in rats will lead to increased levels of IL18 in the hippocampus and behavioral modifications. ${ }^{66}$ Polymorphisms in the IL18 and IL18 receptor genes have also been associated with schizophrenia. ${ }^{67,68}$

The cytokine receptor IL4R binds IL4, which has been referred to as the 'prototypic immunoregulatory cytokine.' ${ }^{69}$ The cytokine can activate T, B and natural killer cells but not monocytes. A meta-analysis of serum cytokine levels shows that IL4 levels are not significantly altered in the serum of patients with schizophrenia as compared to controls, ${ }^{3}$ however, RNA levels of IL4R are significantly increased in the hippocampus of patients with schizophrenia. ${ }^{15} \mathrm{~A}$ recent study has found that in response to sublethal ischemia, IL-4 can be produced and secreted by neurons, resulting in the upregulation of IL4R on microglia and consequently propose this as an endogenous defense mechanism in the brain. $^{70}$ The interleukin 15 (IL15) receptor consists of three subunits, IL2R beta, IL2R gamma and IL15R alpha. ${ }^{71}$ IL15RA encodes the alpha subunit that contributes to the high-affinity binding of IL15, which has a broad range of biological functions as an inflammatory and immune modulator responding to microbial infections and parasites. ${ }^{71} \mathrm{IL}-15$ is upregulated in the serum of first episode, antipsychotic-naive patients with schizophrenia. ${ }^{72,73}$

In addition to these genes common to both collections, we also found genes related to response to virus, such as MX1 (myxovirus (influenza virus) resistance 1 , interferon-inducible) and $M X 2$ (myxovirus (influenza virus) resistance 2 ) and genes related to response to molecules of bacterial origin such as IL6R, TLR2 and STAT1 that were significantly enriched in the S_M16 module. Interleukin 6 (IL6) that binds to IL6R is consistently elevated in the serum of patients with schizophrenia ${ }^{3}$ and the co-expression module, S_M16 was positively correlated with IL6 protein levels in the frontal cortex of the same individuals (Table 1). Toll-like receptor 2 (TLR2) expression is induced in the CP during the acute phase of the LPS mouse model ${ }^{31}$ and genes related to IL-2, IL-4, IL- 6 and IL-10 signaling were also increased in the CP of mice that received LPS once every 2 weeks for 3 months. ${ }^{74}$ We also found a substantial numbers of genes that were common to the coexpression modules of both the LPS mouse model (LPS_3h_M2) and the schizophrenia-associated immune/inflammation response module (S_Co_M16). Recent large scale genome wide association studies have shown the most significant association between schizophrenia and common variants is on the major histocompatibility complex (MHC) region of chromosome $6 .^{75-77}$ This result suggests that abnormal immune/inflammation response may be involved in the etiology of schizophrenia. HLA class I genes such as $H L A-A, H L A-B$ and $H L A-C$ are located in the MHC region and are included in the schizophrenia specific module, S_R_only_M1, from our replication study. MHC class I molecules bind to fragments of viral proteins and present them to the cell surface for recognition by $\mathrm{CD}^{+}{ }^{\mathrm{T}}$ cells. ${ }^{78}$ In addition, the co-expression module, S_M16 was also positively correlated with CRP levels in both the serum and frontal cortex of the same individuals (Table 1). CRP levels can be increased by infection and/or inflammation, ${ }^{79}$ so taken together, these results suggest that a peripheral stimulus, perhaps viral and/or bacterial, may activate the co-expression module related to immune/inflammation responses in the $\mathrm{CP}$ of patients with schizophrenia. Furthermore, these results suggest the possible causal relationship between activated immune/inflammation response-related genes and schizophrenia.

Although antibodies to various infectious agents have been identified in the serum of patients with schizophrenia and are often associated with increased risk for the disorder, ${ }^{80}$ robust hallmarks of neuropathological inflammation in the brain of people with schizophrenia have not been reported. This indicates that if infectious agents are involved in schizophrenia it is likely a mild chronic infection rather than an acute infection. In addition, maternal immune activation (MIA) also may contribute significantly to the increased risk of developing schizophrenia in the offspring, ${ }^{81}$ and it is proposed that once the acute inflammation subsides an abnormal 'immune memory' may continue to be active in the offspring that then interferes with brain development into adulthood. ${ }^{82}$ The abnormal cytokine and CRP levels and the immune/inflammation co-expression module in the CP of people with schizophrenia may all be signatures of this abnormal 'immune memory'. Although animal models of MIA often show an increase in cytokine levels in serum and brain of the adult offspring, ${ }^{83,84}$ other similar studies did not show these effects. ${ }^{85,86}$ However, MIA appears to have a different effect on cytokine levels at different development stages in the offspring (infancy, adolescence and adulthood), and in different brain regions. ${ }^{83}$ The timing of immune activation, the intensity of immune stimulus and the genetic background may also contribute to the differential effects in the offspring. Interestingly, animals that receive immune activation as adults show changes in mRNA expression of immune-related genes in the frontal cortex that reflects changes seen in the frontal cortex of people with schizophrenia. ${ }^{84}$ 
The co-expression module, S_M16 was also positively correlated with cortisol protein levels in the serum and in the frontal cortex of the same individuals (Table 1), indicating that the module could be activated in response to chronic stress, which is known to modulate the immune/inflammation response. ${ }^{87}$ Cortisol is a steroid hormone released in response to stress and levels are elevated in serum ${ }^{88,89}$ and in frontal cortex ${ }^{41}$ of individuals with schizophrenia. Although both human and animal studies show that chronic stress increases inflammatory cytokines in the blood and the brain, ${ }^{87}$ it is also apparent that cytokines such as IL1 and IL6 can act as potent activators of the HPA axis. ${ }^{88,90,91}$ Thus, although chronic stress may be contributing to the activated immune/inflammation module in the $\mathrm{CP}$ of schizophrenia it is likely part of a complex interaction.

A recent genetic study proposed complement component 4 (C4) as a possible candidate gene in the MHC region of chromosome 6, for the pathogenesis of schizophrenia. ${ }^{92}$ C4, located near the strongest peak of association with schizophrenia at the $\mathrm{MHC}$ region, ${ }^{92}$ has two isotypes of the gene, $C 4 A$ and $C 4 B$, in humans. Besides a key role in innate immune response, the complement system is implicated in synaptic pruning. ${ }^{93} \mathrm{C} 4$ protein mediates synaptic plasticity in the mouse model and C4A mRNA expression is significantly higher in individuals with schizophrenia as compared with the unaffected controls. ${ }^{92}$ Three complement genes $C 1 R, C 1 R L$ and $C 3$ were included in the S_M16 module in this study and C1R,C1RL, C1S and C1QL were included in the immune module from the hippocampus RNA-seq data from schizophrenia and controls from our previous study. ${ }^{94}$ These results suggest a possible mechanism for mediating the excess synaptic pruning that may occur in the brain of schizophrenia patients by the activated immune/inflammation related modules of co-expressed genes.

Gene co-expression network analysis is now a standard statistical approach for identifying differentially expressed genes at the systems level when comparing disease cases and controls. ${ }^{34}$ This systems approach may identify gene-gene interactions (that is, connectivity between genes) that contribute to the pathophysiology of the disease. ${ }^{39}$ In this study, we not only performed single-gene comparison and gene co-expression analysis but also performed independent co-expression analysis for cases and controls separately to detect gene-gene interactions that differed between disease cases and unaffected controls. We identified 27 differentially expressed genes in our first datasets and 33 in the replication dataset but only three genes overlapped. In contrast, the co-expression network analysis from the replication dataset yielded results that were more consistent with those from the first dataset. The immune-related module, S-R_M2, from the replication dataset included 84 genes ( 40\%), which were also included in the immune-related module, S M 16, from the first dataset. This indicates that reproducibility of the co-expression network analyses may be higher than the classical single-gene comparison analysis. Although none of the modules were significantly associated with schizophrenia in the separate analysis, one immune-related module was significantly associated with schizophrenia in the analysis of the combined datasets. Thus, a larger sample size may be necessary to detect modules associated with schizophrenia in the network analysis.

The co-expression network shows that genes relating to immune function and inflammation are co-expressed in both cases and controls, in both tissue collections, however, the overall expression of the network seemed to be upregulated in schizophrenia as compared with controls. When we built independent co-expression modules for schizophrenia and controls separately and then compared the co-expression modules for schizophrenia to those for controls, or vice versa, we found that almost all the modules were conserved between the schizophrenia cases and controls indicating that there is generally no difference in the gene-gene interactions between cases and controls. However, there were several co-expression networks that were only weakly conserved in controls in both collections and therefore likely to be unique to schizophrenia, however, these were also enriched for genes related to immune response as well as apoptosis. Thus, although there is a network of immune/ inflammation related genes in the $\mathrm{CP}$ that is responding to stimuli from the periphery in both schizophrenia cases and controls, the response is significantly upregulated in schizophrenia. This upregulated network of genes may then upregulate an additional network of genes related to immune defense and apoptosis that is unique to the $C P$ in the schizophrenia cases.

Genome-wide gene expression profiling studies such as microarray and mRNA sequencing (RNA-Seq) have been widely used as an unbiased approach to identify the pathophysiology of schizophrenia. ${ }^{10-15,95,96}$ Although microarray studies have identified many genes associated with the etiology of schizophrenia, ${ }^{10-13,95,96}$ the results need to be validated using independent methods, such as RT-PCR, because of the high levels of noise in the gene expression measurements. ${ }^{97}$ However, advances in massively parallel sequencing methodology such as RNA-Seq can provide more accurate, sensitive and reliable gene expression data than both microarrays and RT-PCR. ${ }^{98}$ Thus, here we performed a replication and validation study by using RNA-seq on a second independent cohort rather than doing RT-PCR on the same cohort.

In conclusion, the CP of individuals with schizophrenia appears to be responding to peripheral stimuli by upregulating genes related to immune function and inflammation. The significant overlap of co-expression networks found in the CP of schizophrenia patients and of the LPS-treated mouse model indicates that there may be a peripheral challenge from the immune system that is affecting the brain in individuals with schizophrenia. These results also support our previous results that showed that peripheral infection and stress may synergistically underlie the pathophysiology of schizophrenia. ${ }^{15}$ It is apparent in animal models that changes in peripheral immunity can affect higher cognitive brain function, ${ }^{98,99}$ but it remains to be determined exactly how the peripheral immune system can regulate brain function and behavior.

\section{Note}

The data analysis procedures are outlined in Supplementary Figure 2. Gene symbols for each co-expression module are listed in Supplementary Table S30. The top 5000 probes and corresponding gene symbols that were selected from the data from mice $3 \mathrm{~h}$ post LPS treatment and controls are listed in Supplementary Table S31. Both RNA-Seq raw data (FASTQ files), read count data and the protein markers measured in serum and frontal cortex of the individuals from the $\mathrm{AC}$ are publicly available for free download at http://sncid.stanleyresearch.org (SNCID).

\section{CONFLICT OF INTEREST}

The authors declare no conflict of interest.

\section{ACKNOWLEDGMENTS}

$\mathrm{DL}$ and $\mathrm{YH}$ were supported by the Bio-Synergy Research Project (NRF-2012M3A9C4048758) of the Korean Ministry of Science, ICT and Future Planning through the Korean National Research Foundation. We thank all the investigators who generated the original data in the SNCID, and their many collaborators, who made this database possible. We also thank Jonathan Cohen for technical support. We specially thank the Macrogen Company for their technical assistance for RNA-Seq. 


\section{REFERENCES}

1 Drexhage RC, Weigelt K, van Beveren N, Cohen D, Versnel MA, Nolen WA et al. Immune and neuroimmune alterations in mood disorders and schizophrenia. Int Rev Neurobiol 2011; 101: 169-201.

2 Kurian SM, Le-Niculescu H, Patel SD, Bertram D, Davis J, Dike C et al. Identification of blood biomarkers for psychosis using convergent functional genomics. Mol Psychiatry 2011; 16: 37-58.

3 Miller BJ, Buckley P, Seabolt W, Mellor A, Kirkpatrick B. Meta-analysis of cytokine alterations in schizophrenia: clinical status and antipsychotic effects. Biol Psychiatry 2011; 70: 663-671.

4 Weigelt K, Carvalho LA, Drexhage RC, Wijkhuijs A, de Wit H, van Beveren NJ et al. TREM-1 and DAP12 expression in monocytes of patients with severe psychiatric disorders. EGR3, ATF3 and PU.1 as important transcription factors. Brain Behav Immun 2011; 25: 1162-1169.

5 Sainz J, Mata I, Barrera J, Perez-Iglesias R, Varela I, Arranz MJ et al. Inflammatory and immune response genes have significantly altered expression in schizophrenia. Mol Psychiatry 2013; 18: 1056-1057.

6 Gardiner EJ, Cairns MJ, Liu B, Beveridge NJ, Carr V, Kelly B et al. Gene expression analysis reveals schizophrenia-associated dysregulation of immune pathways in peripheral blood mononuclear cells. J Psychiatr Res 2013; 47: 425-437.

7 Soderlund J, Schroder J, Nordin C, Samuelsson M, Walther-Jallow L, Karlsson H et al. Activation of brain interleukin-1beta in schizophrenia. Mol Psychiatry 2009; 14: $1069-1071$.

8 Bechter K, Reiber H, Herzog S, Fuchs D, Tumani H, Maxeiner HG. Cerebrospinal fluid analysis in affective and schizophrenic spectrum disorders: identification of subgroups with immune responses and blood-CSF barrier dysfunction. J Psychiatr Res 2010; 44: 321-330.

9 Maxeiner HG, Rojewski MT, Schmitt A, Tumani H, Bechter K, Schmitt M. Flow cytometric analysis of $\mathrm{T}$ cell subsets in paired samples of cerebrospinal fluid and peripheral blood from patients with neurological and psychiatric disorders. Brain Behav Immun 2009; 23: 134-142.

10 Saetre $P$, Emilsson L, Axelsson E, Kreuger J, Lindholm E, Jazin E. Inflammationrelated genes up-regulated in schizophrenia brains. BMC Psychiatry 2007; 7: 46.

11 Arion D, Unger T, Lewis DA, Levitt $P$, Mirnics $K$. Molecular evidence for increased expression of genes related to immune and chaperone function in the prefrontal cortex in schizophrenia. Biol Psychiatry 2007; 62: 711-721.

12 Shao L, Vawter MP. Shared gene expression alterations in schizophrenia and bipolar disorder. Biol Psychiatry 2008; 64: 89-97.

13 Schmitt A, Leonardi-Essmann F, Durrenberger PF, Parlapani E, Schneider-Axmann $\mathrm{T}$, Spanagel $\mathrm{R}$ et al. Regulation of immune-modulatory genes in left superior temporal cortex of schizophrenia patients: a genome-wide microarray study. World J Biol Psychiatry 2011; 12: 201-215.

14 Fillman SG, Cloonan N, Catts VS, Miller LC, Wong J, McCrossin T et al. Increased inflammatory markers identified in the dorsolateral prefrontal cortex of individuals with schizophrenia. Mol Psychiatry 2013; 18: 206-214.

15 Hwang Y, Kim J, Shin JY, Kim Jl, Seo JS, Webster MJ et al. Gene expression profiling by mRNA sequencing reveals increased expression of immune/inflammationrelated genes in the hippocampus of individuals with schizophrenia. Translat Psychiatry 2013; 3: e321.

16 Fillman SG, Sinclair D, Fung SJ, Webster MJ, Shannon Weickert C. Markers of inflammation and stress distinguish subsets of individuals with schizophrenia and bipolar disorder. Translat Psychiatry 2014; 4: e365.

17 Busse S, Busse M, Schiltz K, Bielau H, Gos T, Brisch R et al. Different distribution patterns of lymphocytes and microglia in the hippocampus of patients with residual versus paranoid schizophrenia: further evidence for disease courserelated immune alterations? Brain Behav Immun 2012; 26: 1273-1279.

18 Schnieder TP, Dwork AJ. Searching for neuropathology: gliosis in schizophrenia. Biol Psychiatry 2011; 69: 134-139.

19 Harris LW, Wayland M, Lan M, Ryan M, Giger T, Lockstone H et al. The cerebral microvasculature in schizophrenia: a laser capture microdissection study. PLOS One 2008; 3: e3964.

20 Uranova NA, Zimina IS, Vikhreva OV, Krukov NO, Rachmanova VI, Orlovskaya DD. Ultrastructural damage of capillaries in the neocortex in schizophrenia. World J Biol Psychiatry 2010; 11: 567-578.

21 Redzic ZB, Segal MB. The structure of the choroid plexus and the physiology of the choroid plexus epithelium. Adv Drug Deliv Rev 2004; 56: 1695-1716.

22 Marques F, Sousa JC. The choroid plexus is modulated by various peripheral stimuli: implications to diseases of the central nervous system. Front Cell Neurosci 2015; 9: 136.

23 Quan N, Mhlanga JD, Whiteside MB, McCoy AN, Kristensson K, Herkenham M. Chronic overexpression of proinflammatory cytokines and histopathology in the brains of rats infected with Trypanosoma brucei. J Comp Neurol 1999; 414: 114-130.

24 Pron B, Taha MK, Rambaud C, Fournet JC, Pattey N, Monnet JP et al. Interaction of Neisseria maningitidis with the components of the blood-brain barrier correlates with an increased expression of PilC. J Infect Dis 1997; 176: 1285-1292.
25 Falangola MF, Hanly A, Galvao-Castro B, Petito CK. HIV infection of human choroid plexus: a possible mechanism of viral entry into the CNS. J Neuropathol Exp Neurol 1995; 54: 497-503.

26 Sathyanesan M, Girgenti MJ, Banasr M, Stone K, Bruce C, Guilchicek E et al. A molecular characterization of the choroid plexus and stress-induced gene regulation. Transl Psychiatry 2012; 2: e139.

27 Newton SS, Collier EF, Hunsberger J, Adams D, Terwilliger R, Selvanayagam E et al. Gene profile of electroconvulsive seizures: induction of neurotrophic and angiogenic factors. J Neurosci 2003; 23: 10841-10851.

28 Trapnell C, Pachter L, Salzberg SL. TopHat: discovering splice junctions with RNA-Seq. Bioinformatics 2009; 25: 1105-1111.

29 Anders S, Pyl PT, Huber W. HTSeq-a Python framework to work with highthroughput sequencing data. Bioinformatics 2015; 31: 166-169.

30 Robinson MD, McCarthy DJ, Smyth GK. edgeR: a Bioconductor package for differential expression analysis of digital gene expression data. Bioinformatics 2010; 26: 139-140.

31 Marques F, Sousa JC, Coppola G, Falcao AM, Rodrigues AJ, Geschwind DH et al. Kinetic profile of the transcriptome changes induced in the choroid plexus by peripheral inflammation. J Cereb Blood Flow Metab 2009; 29: 921-932.

32 Oldham MC, Horvath S, Geschwind DH. Conservation and evolution of gene coexpression networks in human and chimpanzee brains. Proc Natl Acad Sci USA 2006; 103: 17973-17978.

33 Miller JA, Oldham MC, Geschwind DH. A systems level analysis of transcriptional changes in Alzheimer's disease and normal aging. J Neurosci 2008; 28: 1410-1420.

34 Langfelder $\mathrm{P}$, Horvath S. WGCNA: an R package for weighted correlation network analysis. BMC Bioinform 2008; 9: 559.

35 Leek JT, Storey JD. Capturing heterogeneity in gene expression studies by surrogate variable analysis. PLoS Genet 2007; 3: 1724-1735.

36 Zhang B, Horvath S. A general framework for weighted gene co-expression network analysis. Stat Appl Genet Mol Biol 2005; 4: Article17.

37 Yoder PJ, Blackford JU, Waller NG, Kim G. Enhancing power while controlling family-wise error: an illustration of the issues using electrocortical studies. J Clin Exp Neuropsychol 2004; 26: 320-331.

$38 \mathrm{Hu}$ Z, Mellor J, Wu J, DeLisi C. VisANT: an online visualization and analysis tool for biological interaction data. BMC Bioinform 2004; 5: 17.

39 Langfelder P, Luo R, Oldham MC, Horvath S. Is my network module preserved and reproducible? PLoS Comput Biol 2011; 7: e1001057.

40 Shannon P, Markiel A, Ozier O, Baliga NS, Wang JT, Ramage D et al. Cytoscape: a software environment for integrated models of biomolecular interaction networks. Genome Res 2003; 13: 2498-2504.

41 Harris LW, Pietsch S, Cheng TM, Schwarz E, Guest PC, Bahn S. Comparison of peripheral and central schizophrenia biomarker profiles. PLoS One 2012; 7: e46368.

42 Dennis G Jr, Sherman BT, Hosack DA, Yang J, Gao W, Lane HC et al. DAVID: database for annotation, visualization, and integrated discovery. Genome Biol 2003; 4: P3.

43 Eden E, Navon R, Steinfeld I, Lipson D, Yakhini Z. GOrilla: a tool for discovery and visualization of enriched GO terms in ranked gene lists. BMC Bioinform 2009; 10: 48.

44 O'Donnell LC, Druhan LJ, Avalos BR. Molecular characterization and expression analysis of leucine-rich alpha2-glycoprotein, a novel marker of granulocytic differentiation. J Leukoc Biol 2002; 72: 478-485.

45 Tanaka Y, Matsumoto I, Iwanami K, Inoue A, Umeda N, Tanaka Y et al. Sixtransmembrane epithelial antigen of prostate 4 (STEAP4) is expressed on monocytes/neutrophils, and is regulated by TNF antagonist in patients with rheumatoid arthritis. Clin Exp Rheumatol 2012; 30: 99-102.

46 Tanaka $\mathrm{Y}$, Matsumoto I, Iwanami $\mathrm{K}$, Inoue A, Minami R, Umeda N et al. Sixtransmembrane epithelial antigen of prostate4 (STEAP4) is a tumor necrosis factor alpha-induced protein that regulates IL-6, IL-8, and cell proliferation in synovium from patients with rheumatoid arthritis. Mod Rheumatol 2012; 22: 128-136.

47 Liddelow SA. Development of the choroid plexus and blood-CSF barrier. Front Neurosci 2015; 9: 32

48 Carter CS, Bullmore ET, Harrison P. Is there a flame in the brain in psychosis? Biol Psychiatry 2014; 75: 258-259.

49 Logotheti M, Papadodima O, Venizelos N, Chatziioannou A, Kolisis F. A comparative genomic study in schizophrenic and in bipolar disorder patients, based on microarray expression profiling meta-analysis. Sci World J 2013; 2013: 685917.

50 Mistry M, Gillis J, Pavlidis P. Genome-wide expression profiling of schizophrenia using a large combined cohort. Mol Psychiatry 2013; 18: 215-225.

51 Bergon A, Belzeaux R, Comte M, Pelletier F, Herve M, Gardiner EJ et al. CX3CR1 is dysregulated in blood and brain from schizophrenia patients. Schizophr Res 2015; 168: 434-443.

52 Huang KC, Yang KC, Lin H, Tsao Tsun-Hui T, Lee WK, Lee SA et al. Analysis of schizophrenia and hepatocellular carcinoma genetic network with corresponding modularity and pathways: novel insights to the immune system. BMC Genomics 2013; 14: S10.

53 Gilmore $\mathrm{JH}$, Bouldin TW. Analysis of ependymal abnormalities in subjects with schizophrenia, bipolar disorder, and depression. Schizophr Res 2002; 57: 267-271. 
54 Marinescu I, Udristoiu I, Marinescu D. Choroid plexus calcification: clinical, neuroimaging and histopathological correlations in schizophrenia. Rom J Morphol Embryol 2013; 54: 365-369.

55 Chelbi ST, Wilson ML, Veillard AC, Ingles SA, Zhang J, Mondon F et al. Genetic and epigenetic mechanisms collaborate to control SERPINA3 expression and its association with placental diseases. Hum Mol Genet 2012; 21: 1968-1978.

56 Trepanier MO, Hopperton KE, Mizrahi R, Mechawar N, Bazinet RP.. Postmortem evidence of cerebral inflammation in schizophrenia: a systematic review. Mol Psychiatry 2016; 21: 1009-1026.

57 Aman MJ, Migone TS, Sasaki A, Ascherman DP, Zhu M, Soldaini E et al. CIS associates with the interleukin-2 receptor beta chain and inhibits interleukin-2dependent signaling. J Biol Chem 1999; 274: 30266-30272.

58 Khor CC, Vannberg FO, Chapman SJ, Guo H, Wong SH, Walley AJ et al. CISH and susceptibility to infectious diseases. N Engl J Med 2010; 362: 2092-2101.

59 Dinarello CA. Biologic basis for interleukin-1 in disease. Blood 1996; 87: 2095-2147.

60 Gracie JA, Forsey RJ, Chan WL, Gilmour A, Leung BP, Greer MR et al. A proinflammatory role for IL-18 in rheumatoid arthritis. J Clin Invest 1999; 104: 1393-1401.

61 Xiu MH, Chen da C, Wang D, Zhang K, Dong A, Tang W et al. Elevated interleukin18 serum levels in chronic schizophrenia: association with psychopathology. $J$ Psychiatr Res 2012; 46: 1093-1098.

62 Palladino I, Salani F, Ciaramella A, Rubino IA, Caltagirone C, Fagioli S et al. Elevated levels of circulating IL-18BP and perturbed regulation of IL-18 in schizophrenia. $J$ Neuroinflamm 2012; 9: 206.

63 Zhang XY, Tang W, Xiu MH, Chen da C, Yang FD, Tan YL et al. Interleukin 18 and cognitive impairment in first episode and drug naive schizophrenia versus healthy controls. Brain Behav Immun 2013; 32: 105-111.

64 Al-Hakeim HK, Al-Rammahi DA, Al-Dujaili AH. IL-6, IL-18, sIL-2R, and TNFalpha proinflammatory markers in depression and schizophrenia patients who are free of overt inflammation. J Affect Disorders 2015; 182: 106-114.

65 Bossu P, Piras F, Palladino I, lorio M, Salani F, Ciaramella A et al. Hippocampal volume and depressive symptoms are linked to serum IL-18 in schizophrenia. Neurol Neuroimmunol Neuroinflamm 2015; 2: e111.

66 Bossu P, Cutuli D, Palladino I, Caporali P, Angelucci F, Laricchiuta D et al. A single intraperitoneal injection of endotoxin in rats induces long-lasting modifications in behavior and brain protein levels of TNF-alpha and IL-18. J Neuroinflamm 2012; 9: 101.

67 Shirts $\mathrm{BH}$, Wood J, Yolken RH, Nimgaonkar VL. Comprehensive evaluation of positional candidates in the IL-18 pathway reveals suggestive associations with schizophrenia and herpes virus seropositivity. Am J Med Genet 2008; 147: 343-350.

68 Liu J, Liu J, Zhou Y, Li S, Li Y, Song X et al. Association between promoter variants of interleukin-18 and schizophrenia in a Han Chinese population. DNA Cell Biol 2011; 30: 913-917.

69 Paul WE. Interleukin-4: a prototypic immunoregulatory lymphokine. Blood 1991; 77: $1859-1870$.

70 Zhao X, Wang H, Sun G, Zhang J, Edwards NJ, Aronowski J. Neuronal interleukin-4 as a modulator of microglial pathways and ischemic brain damage. $J$ Neurosci 2015; 35: 11281-11291.

71 Perera PY, Lichy JH, Waldmann TA, Perera LP. The role of interleukin-15 in inflammation and immune responses to infection: implications for its therapeutic use. Microbes Infect 2012; 14: 247-261.

72 Ramsey JM, Schwarz E, Guest PC, van Beveren NJ, Leweke FM, Rothermundt M et al. Distinct molecular phenotypes in male and female schizophrenia patients. PLoS One 2013; 8: e78729.

73 de Witte L, Tomasik J, Schwarz E, Guest PC, Rahmoune H, Kahn RS et al. Cytokine alterations in first-episode schizophrenia patients before and after antipsychotic treatment. Schizophr Res 2014; 154: 23-29.

74 Marques F, Sousa JC, Coppola G, Geschwind DH, Sousa N, Palha JA et al. The choroid plexus response to a repeated peripheral inflammatory stimulus. $B M C$ Neurosci 2009; 10: 135.

75 Purcell SM, Wray NR, Stone JL, Visscher PM, O'Donovan MC, Sullivan PF et al. Common polygenic variation contributes to risk of schizophrenia and bipolar disorder. Nature 2009; 460: 748-752.

76 Stefansson H, Ophoff RA, Steinberg S, Andreassen OA, Cichon S, Rujescu D et al. Common variants conferring risk of schizophrenia. Nature 2009; 460: 744-747.

77 Schizophrenia Working Group of the Psychiatric Genomics Consortium. Biological insights from 108 schizophrenia-associated genetic loci. Nature 2014; 511: 421-427.

78 Neefjes J, Jongsma ML, Paul P, Bakke O. Towards a systems understanding of MHC class I and MHC class II antigen presentation. Nat Rev Immunol 2011; 11: 823-836.
79 Young B, Gleeson M, Cripps AW. C-reactive protein: a critical review. Pathology 1991; 23: 118-124.

80 Arias I, Sorlozano A, Villegas E, de Dios Luna J, McKenney K, Cervilla J et al. Infectious agents associated with schizophrenia: a meta-analysis. Schizophr Res 2012; 136: 128-136.

81 Brown AS, Derkits EJ. Prenatal infection and schizophrenia: a review of epidemiologic and translational studies. Am J Psychiatry 2010; 167: 261-280.

82 Horvath S, Mirnics K. Immune system disturbances in schizophrenia. Biol Psychiatry 2014; 75: 316-323.

83 Borrell J, Vela JM, Arevalo-Martin A, Molina-Holgado E, Guaza C. Prenatal immune challenge disrupts sensorimotor gating in adult rats. Implications for the etiopathogenesis of schizophrenia. Neuropsychopharmacology 2002; 26: 204-215.

84 Garay PA, Hsiao EY, Patterson PH, McAllister AK. Maternal immune activation causes age- and region-specific changes in brain cytokines in offspring throughout development. Brain Behav Immun 2013; 31: 54-68.

85 Volk DW, Chitrapu A, Edelson JR, Roman KM, Moroco AE, Lewis DA. Molecular mechanisms and timing of cortical immune activation in schizophrenia. Am J Psychiatry 2015; 172: 1112-1121.

86 Meyer U, Murray PJ, Urwyler A, Yee BK, Schedlowski M, Feldon J. Adult behavioral and pharmacological dysfunctions following disruption of the fetal brain balance between pro-inflammatory and IL-10-mediated anti-inflammatory signaling. Mol Psychiatry 2008; 13: 208-221.

87 Glaser R, Kiecolt-Glaser JK. Stress-induced immune dysfunction: implications for health. Nat Rev Immunol 2005; 5: 243-251.

88 Zhang XY, Zhou DF, Cao LY, Wu GY, Shen YC. Cortisol and cytokines in chronic and treatment-resistant patients with schizophrenia: association with psychopathology and response to antipsychotics. Neuropsychopharmacology 2005; 30: 1532-1538.

89 Mondelli V, Ciufolini S, Belvederi Murri M, Bonaccorso S, Di Forti M, Giordano A et al. Cortisol and inflammatory biomarkers predict poor treatment response in first episode psychosis. Schizophr Bull 2015; 41: 1162-1170.

90 Altamura AC, Boin F, Maes M. HPA axis and cytokines dysregulation in schizophrenia: potential implications for the antipsychotic treatment. Eur Neuropsychopharmacol 1999; 10: 1-4.

91 Wang J, Dunn AJ. The role of interleukin-6 in the activation of the hypothalamopituitary-adrenocortical axis and brain indoleamines by endotoxin and interleukin-1 beta. Brain Res 1999; 815: 337-348.

92 Sekar A, Bialas AR, de Rivera H, Davis A, Hammond TR, Kamitaki N et al. Schizophrenia risk from complex variation of complement component 4. Nature 2016; 530: 177-183.

93 Stevens B, Allen NJ, Vazquez LE, Howell GR, Christopherson KS, Nouri N et al. The classical complement cascade mediates CNS synapse elimination. Cell 2007; 131 1164-1178.

94 Kim S, Hwang Y, Webster MJ, Lee D. Differential activation of immune/inflammatory response-related co-expression modules in the hippocampus across the major psychiatric disorders. Mol Psychiatry 2016; 21: 376-385.

95 Middleton FA, Mirnics K, Pierri JN, Lewis DA, Levitt P. Gene expression profiling reveals alterations of specific metabolic pathways in schizophrenia. J Neurosci 2002; 22: 2718-2729.

96 Arion D, Horvath S, Lewis DA, Mirnics K. Infragranular gene expression disturbances in the prefrontal cortex in schizophrenia: signature of altered neural development? Neurobiol Dis 2010; 37: 738-746.

97 Leek JT, Scharpf RB, Bravo HC, Simcha D, Langmead B, Johnson WE et al. Tackling the widespread and critical impact of batch effects in high-throughput data. Nat Rev Genet 2010; 11: 733-739.

98 Marioni JC, Mason CE, Mane SM, Stephens M, Gilad Y. RNA-seq: an assessment of technical reproducibility and comparison with gene expression arrays. Genome Res 2008; 18: 1509-1517.

99 Schwartz M, Kipnis J. A conceptual revolution in the relationships between the brain and immunity. Brain Behav Immun 2011; 25: 817-819.

This work is licensed under a Creative Commons Attribution 4.0 nternational License. The images or other third party material in this article are included in the article's Creative Commons license, unless indicated otherwise in the credit line; if the material is not included under the Creative Commons license, users will need to obtain permission from the license holder to reproduce the material. To view a copy of this license, visit http://creativecommons.org/licenses/ by/4.0/

(c) The Author(s) 2016

upplementary Information accompanies the paper on the Translational Psychiatry website (http://www.nature.com/tp) 